\title{
Enhancement effect of pre-reacted glass on strength of glass-ionomer cement
}

\author{
Naruporn MONMATURAPOJ, Wiwaporn SOODSAWANG and Siriporn TANODEKAEW \\ National Metal and Materials Technology Center (MTEC), 114 Thailand Science Park, Pathumthani 12120, Thailand \\ Corresponding author, Naruporn MONMATURAPOJ; E-mail: narupork@mtec.or.th
}

\begin{abstract}
In this paper, we report on the enhanced strength of glass ionomer cement (GIC) by using the process of pre acid-base reaction and spray drying in glass preparation. The pre acid-base reaction was induced by prior mixing of the glass powder with poly(alkenoic acid). The weight ratios of glass powder to poly(alkenoic acid) were varied to investigate the extent of the pre acid-base reaction of the glass. The effect of the spray drying process which produced spherical glass particles on cement strength was also studied and discussed. The results show that adding $2 \%$-wt of poly(alkenoic acid) liquid in the pre-reacted step improved cement strength. GICs prepared using a mixture of pre-reacted glass with both spherical and irregular powders at 60:40 by weight exhibited the highest compressive strength at $138.64 \pm 7.73 \mathrm{MPa}$. It was concluded that glass ionomer cements containing pre-reacted glass with mixed glass morphology using both spherical and irregular forms are promising as restorative dental materials with improved mechanical properties and handling characteristics.
\end{abstract}

Keywords: Glass ionomer, Ionomer cement, Silicate glass, Dental material, Pre-reacted glass

\section{INTRODUCTION}

Conventional glass ionomer cements (GICs) were firstly described by Wilson and Kent ${ }^{1,2}$ and have been well known in dental material science for nearly 40 years, particularly as a restorative material. Originally, GICs were composed of an aqueous poly(alkenoic acid) such as poly(acrylic acid) which underwent an acid-base reaction with a powder of calcium fluoroaluminosilicate glass ${ }^{3-7)}$. Conventional GICs, were mainly of interest due to their superior advantages, for example, adhesion to tooth structure, slow release of fluoride, good biocompatibility and having a color close to that of enamel ${ }^{1,8}$. On the other hand, they were restricted by the sensitivity to moisture and set as relatively opaque cements ${ }^{7}$. Therefore, the GICs underwent considerable continuing development to improve their properties, in particular, strength and toughness for dentistry and surgical applications.

The early modification of GICs to improve the mechanical properties by blending glass powder with dried polymer powder, and activating cement formation via water addition was studied by Prosser et al.9. . The mixing of spherical and non-spherical glass powders in combination with varying the $\mathrm{F} / \mathrm{Si}$ ratios was also suggested to enhance the cement strength ${ }^{10)}$. Silanization by treating the glass powder with the silanol group was also performed for this intention ${ }^{11}$. The influence of glass compositions such as the alumina to silica ratio ${ }^{12)}$, amount of fluoride ${ }^{13)}$, zinc and metal oxides incorporating glass ionomer2), for example, on the properties of GICs was also investigated. Not only were efforts made to enhance the mechanical properties of GICs, but fluidity, handling characteristics and workability were also investigated by mixing the glass powder with fluorocomplex salt ${ }^{14)}$. The introduction of hydroxyapatite and fluoroapatite, as for the biological aspect, into GICs was also studied with the aim of improving cement strength $^{15}$. Additionally, a process for producing spherical glass powder was developed ${ }^{6)}$ using flame spraying and inductively coupled radio frequency plasma spraying techniques. The formation of spherical particles of glass powder using the spray drying method was also applied to increase cement strength ${ }^{16}$. Spherical particles reduced stress arising at the sharp edges of irregular glass particles, aided mixing due to its flow ability, and in turn, improved the strength of GICs ${ }^{16,17)}$. A previous study by Crisp et al. ${ }^{18)}$ investigated the reaction of glass ionomer cements during setting using the technique of attenuated total reflectance (ATR).

Several investigations reported the improved mechanical strength using pre-reacted glass ionomer fillers. These included the improved mechanical properties and fluoride release of resin composites using a combined filler of pre-reacted glass ionomer and ceramic whiskers ${ }^{19)}$, and the superior adhesiveness of dental adhesives containing pre-reacted glass ionomer fillers ${ }^{20-22)}$. The powder and liquid components of GICs were mixed to undergo acid-base reaction, followed by milling to obtain a pre-reacted glass powder. In this study, a pre-reacted glass was used with a hypothesis of improving mechanical strength by enhancing cement formation. A spray-drying technique was employed to produce simultaneously the pre-reacted glass in spherical form. The effects of the pre-reacted glass and sphericalshaped powder on mechanical strength of the conventional glass ionomer cements were investigated.

\section{MATERIALS AND METHODS}

\section{Glass preparation}

A schematic of glass preparation is presented in Fig. 1 . The glass composition illustrated in Table 1 was modified 


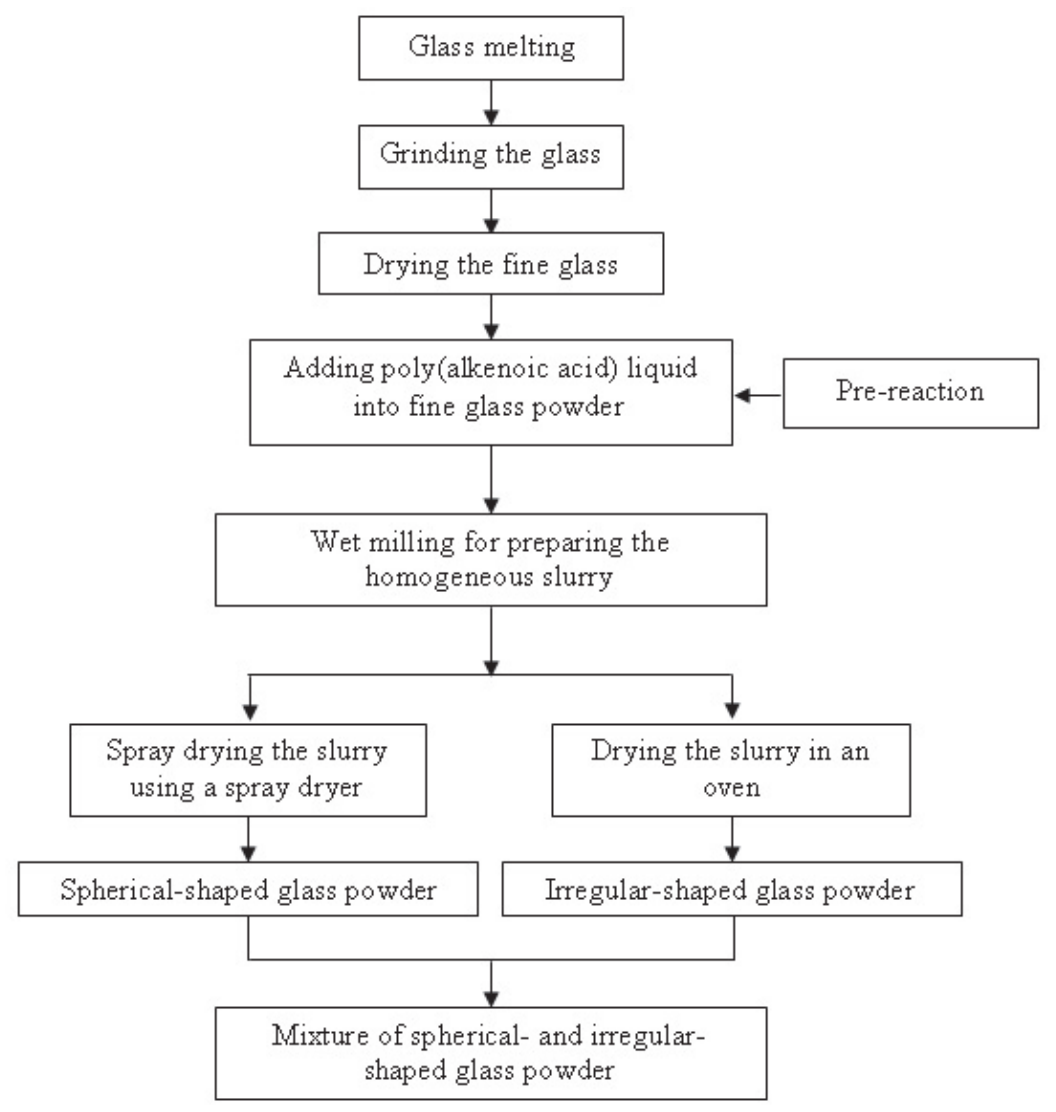

Fig. 1 Schematic of glass preparation.

Table 1 Glass composition

\begin{tabular}{lcc}
\hline Oxides & $\begin{array}{l}\text { Pre-melted glass } \\
\text { compositions (\%-wt) }\end{array}$ & $\begin{array}{c}\text { Post-melted glass } \\
\text { compositions (\%-wt) }\end{array}$ \\
\hline $\mathrm{SiO}_{2}$ & 22.24 & 23.16 \\
$\mathrm{Al}_{2} \mathrm{O}_{3}$ & 20.59 & 25.46 \\
$\mathrm{P}_{2} \mathrm{O}_{5}$ & 12.77 & 12.02 \\
$\mathrm{CaO}$ & - & 1.05 \\
$\mathrm{SrO}$ & 22.50 & 19.71 \\
$\mathrm{CaF}$ & 14.16 & 11.56 \\
$\mathrm{ZrO}_{2}$ & 6.93 & 4.45 \\
$\mathrm{Total}_{2}$ & 99.19 & 97.41 \\
$\mathrm{Al}_{2} \mathrm{O}_{3}: \mathrm{SiO}_{2}$ & 0.93 & 1.10 \\
\hline
\end{tabular}

Table 2 Compressive strength of glass ionomer cements made from the pre-reated glass using different amounts of Fuji II liquid

\begin{tabular}{cc}
\hline Fuji II liquid (\%-wt) & $\begin{array}{c}\text { Mean compressive strength } \\
(\mathrm{MPa})\end{array}$ \\
\hline 0 & $100.08 \pm 15.58$ \\
2 & $121.38 \pm 8.15$ \\
5 & $114.37 \pm 9.39$ \\
7 & $98.15 \pm 14.67$ \\
\hline
\end{tabular}

from the composition reported by Hurrell-Gillingham et $a l .^{23)}$. The glass was melted at $1450^{\circ} \mathrm{C}$ for $2 \mathrm{~h}$ to make glass frit and then ground to attain a mean particle size of about $5 \mu \mathrm{m}$, as measured by Mastersizer 2000 (Malvern instruments, Worcestershire, UK). To prepare the pre-reacted glass, the glass powder was mixed with Fuji II liquid (GC Corporation, Tokyo, Japan) in different portions as shown in Table 2. The mixture was then subjected to ball mill for $3 \mathrm{~h}$ to achieve homogeneous slurry. A laboratory spray dryer (Büchi Mini Spray Dryer, B-290, Büchi Corporation, Flawil, Switzerland) was applied to spray the slurry using an inlet temperature of drying air at $200^{\circ} \mathrm{C}$ (outlet temperature $\approx 105-120^{\circ} \mathrm{C}$ ). The pre-reacted glass before and after spray-drying was mixed at weight ratios of 75:25, 60:40, 50:50, 40:60 and 25:75 in order to investigate the effect of the spray-drying process on the strength of the glass ionomer cements.

\section{Glass characterization}

X-ray diffraction (XRD)

An X-ray diffractometer (JDX 3530, Japan Electron Optics Laboratory Company Limited, Tokyo, Japan) was used to identify phase present in the glass, using $\mathrm{CuK}_{\alpha}$ radiation $(\lambda=1.5406 \AA$ ) operating at $30 \mathrm{kV}$ and $40 \mathrm{~mA}$. Scans were performed on a powder sample with a step size of $0.02^{\circ}$ over an angular range $2 \theta=10-70^{\circ}$ using a scanning speed of $2 \% \mathrm{~min}$. The spectra were analyzed 
using JADE v.6.5 software and JCPDS cards.

Wave length dispersive X-ray fluorescence (WDXRF) The composition of the glass after melting was analyzed by WDXRF (PW 2404, Philips Company, Amsterdam, Netherlands). A sample was prepared by pressing the mixture of glass powder and boric acid $\left(\mathrm{H}_{3} \mathrm{BO}_{3}\right)$ powder to produce a pellet, which was then irradiated with high-energy primary X-ray photons. The weight percentage of the oxides was calculated.

Fourier transform infrared spectroscopy (FTIR)

FTIR spectra were recorded with a FTIR spectrometer (Perkin Elmer System 2000, Perkin Elmer Public Company, UK) to examine the occurrence of pre-reaction of the glass powder. Pellet samples were prepared by mixing and grinding a small amount of glass powder with dried spectroscopic grade $\mathrm{KBr}$ powder and then pressing the mixture to form pellets. The spectra obtained were in the range of $4,000-400 \mathrm{~cm}^{-1}$, averaging 20 scans with the resolution of $4 \mathrm{~cm}^{-1}$.

Scanning electron microscopy (SEM) and particle size analysis

The morphology of the glass particles was determined by SEM (JSM-5410, Japan Electron Optics Laboratory, Tokyo, Japan) operated at $15 \mathrm{keV}$. The samples were prepared by dispersing a thin layer of the glass powders on carbon tape and coated with gold prior to analysis.

The particle size of the glass powder before and after spray drying was analyzed using, respectively, laser diffraction technique (Mastersizer 2000, Malvern instruments, UK) and an optical microscope equipped with Image-Pro Plus program version 5.0.

\section{Cement fabrication and compressive strength} measurement

The glass powder was mixed with Fuji II liquid, using the powder to liquid ratio as cited in the product instruction - 1 scoop of powder: 1 drop of liquid. Cylindrical specimens with dimensions of $6 \mathrm{~mm}$ height $\times 4 \mathrm{~mm}$ diameter were produced by placing the mixed materials into stainless steel molds. After being stored in an incubator at $37^{\circ} \mathrm{C} \pm 2^{\circ} \mathrm{C}$ for $1 \mathrm{~h}$, the specimens were gently removed from the molds, immersed in deionized water and incubated at $37^{\circ} \mathrm{C} \pm 2^{\circ} \mathrm{C}$ for $23 \mathrm{~h}$. The Universal Testing Machine (Instron Model 55R4502, Instron Corporation, Canton, MA, USA) was used to measure the cement compressive strength (CS) at a cross-head speed of $0.75 \mathrm{~mm} / \mathrm{min}$. The CS was determined using the following equation: $\mathrm{CS}=4 \mathrm{P} / \pi \mathrm{D}^{2}$, where $\mathrm{P}$ is the maximum applied load $(\mathrm{N})$ and $\mathrm{D}$ is a diameter of the specimen (mm) according to ISO 9917-124).

\section{RESULTS}

\section{Glass preparation}

Figure 2 shows the XRD pattern of the glass produced. The presence of an amorphous structure with the minor crystalline formation of baddeleyite $\left(\mathrm{ZrO}_{2}\right.$, JCPDS No.
37-1484) appeared in the XRD pattern of the glass.

Table 1 presents the glass composition and $\mathrm{Al}_{2} \mathrm{O}_{3}: \mathrm{SiO}_{2}$ ratio determined by XRF after melting. $\mathrm{A}$ small increment of the $\mathrm{Al}_{2} \mathrm{O}_{3}: \mathrm{SiO}_{2}$ ratio was observed in post-melting caused by the contamination of alumina from alumina crucible during melting. A reduction in the fluoride content of the post-melted glass was also observed due to the volatilization of $\mathrm{SiF}_{4}$ during melting.

\section{Glass characterization}

Principally, GICs sets via a neutralization of acid-base reaction between glass powder and poly(alkenoic acid) liquid. After mixing the powder-liquid components,

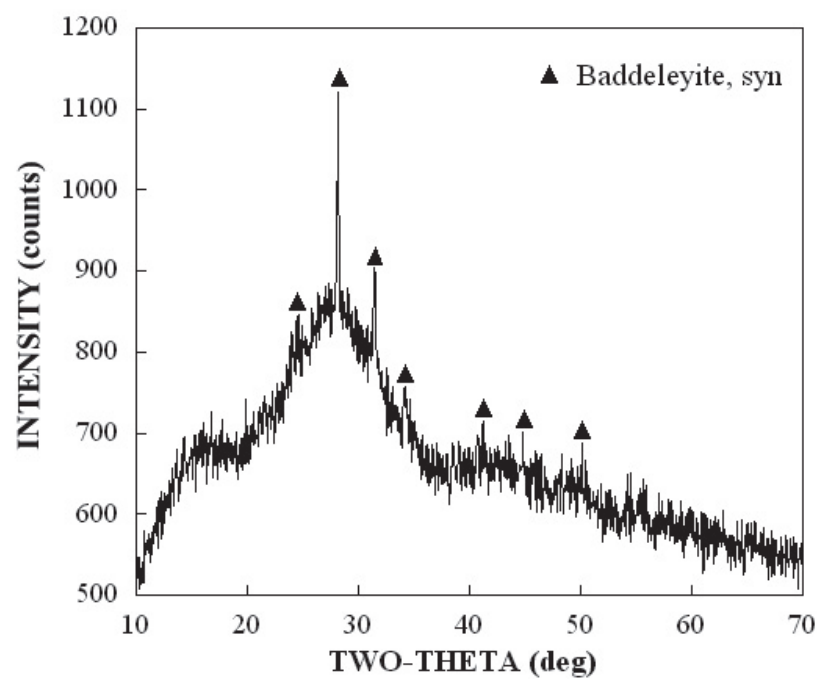

Fig. 2 XRD pattern of the glass studied.

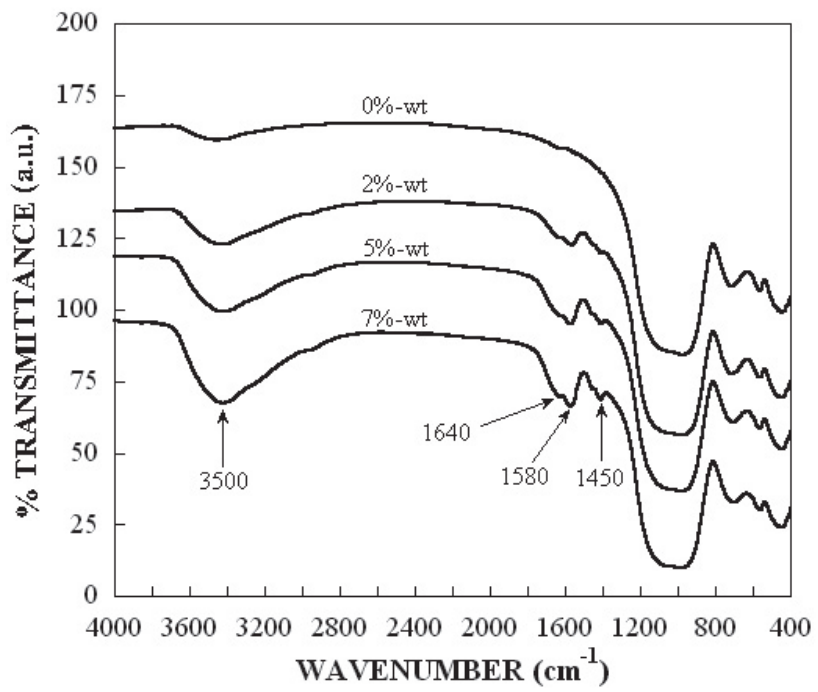

Fig. 3 FTIR spectra of pre-reacted glass produced using varying amounts of poly(alkenoic acid). 

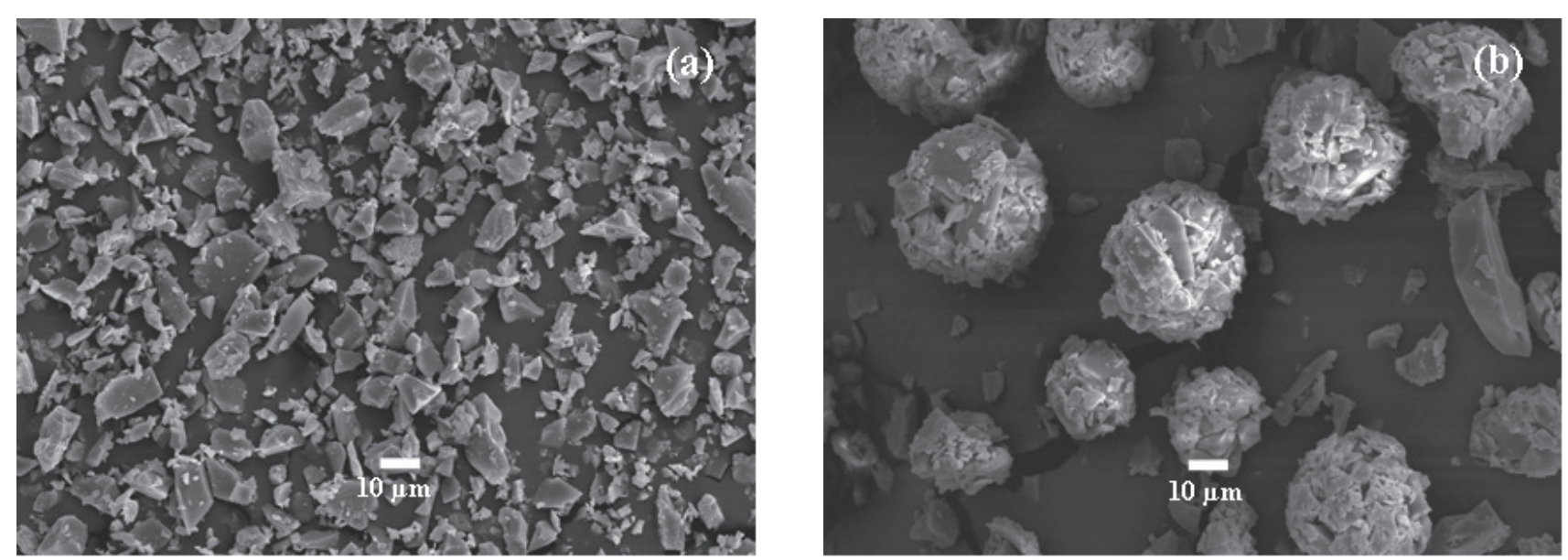

Fig. 4 SEM images of glass powder before a) and after b) spray drying.

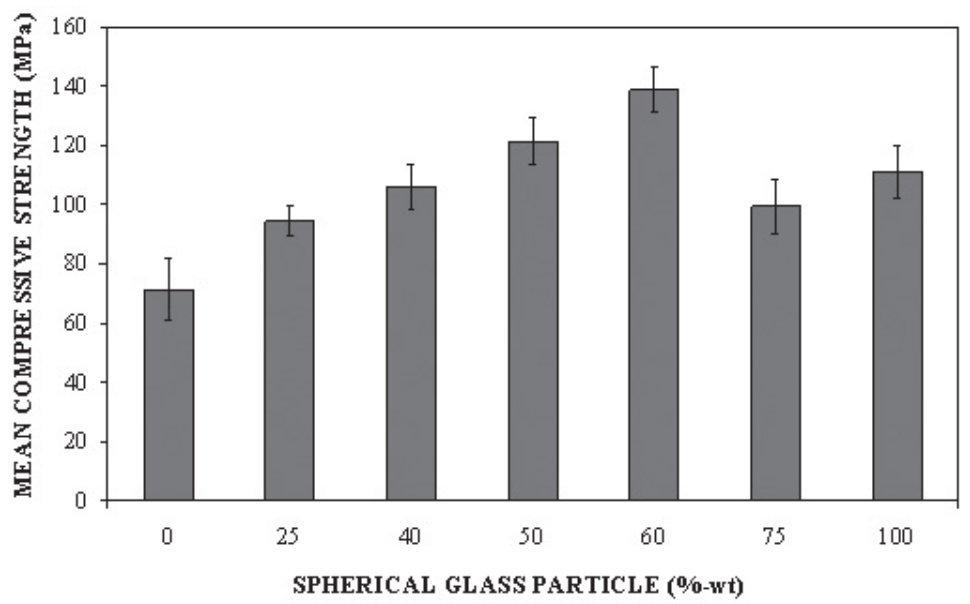

Fig. 5 Compressive strengths of glass ionomer cements prepared from the pre-reacted glass with varying amounts of spherical powder.

acidic degradation of the glass powder results in the release of a cement-forming metal ion $\left(\mathrm{Ca}^{2+}\right.$ and $\left.\mathrm{Al}^{3+}\right)$, and then the generation of metal carboxylate salt by cross-linking ionized carboxylic acid groups in the polymer chain ${ }^{25)}$, leading to the setting of cement by gelation $^{2,7)}$. Therefore, the pre-reacted or pre acid-base reaction refers to the situation in which the carboxylate salt forms in the glass powder.

The FTIR spectra of the pre-reacted glass compared to the original glass powder are shown in Fig. 3. The pre acid-base reaction partly took place in the glass powder as evidenced from new broad peaks at 1,580 and 1,450 $\mathrm{cm}^{-1}$, corresponding to antisymmetrical and symmetrical stretching of carboxylate salt, respectively ${ }^{18)}$. These peaks were found to increase in intensity as the amount of poly(alkenoic acid) increased. There were also peaks appearing at $1,640 \mathrm{~cm}^{-1}$ and $3,500 \mathrm{~cm}^{-1}$, due to water absorption.
Table 2 presents the influence of the extent of pre acid-base reaction on the compressive strength of GICs. A higher compressive strength was expected for a higher extent of pre acid-base reaction, however, the opposite strength results were observed. The strength was found highest for the pre-reacted glass produced from $2 \%$-wt Fuji II liquid and weakened as the extent of the acid-base reaction increased.

Figure 4 is the SEM images illustrating the morphologies of the glass powder before and after spray drying. The glass before spray drying appeared in an irregular shape with a sharp particle edge, having an average particle size of around $5 \mu \mathrm{m}$. The sharp edge of the particle was the result of the grinding by ball milling. After spray drying, the glass formed a loosely agglomerated spherical particle having a mean particle size of about $25 \mu \mathrm{m}$.

The effect of particle shape on cement strength was 
observed using the glass mixture with the irregularshaped particles and the spherical particles obtained before and after spray drying, respectively. The compressive strength of the glass powder with different spherical and irregular-shaped particle ratios is presented in Fig. 5. The results revealed an increase in cement strength if the spherical glass that eased cement handling was used, as expected. The strength was found to increase from $71.33 \pm 10.43 \mathrm{MPa}$ for the irregularshaped powder to the ultimate strength at $138.64 \pm 7.73$ MPa when the combination of spherical- and irregularshaped glass particles at a weight ratio of 60:40 was applied. When the amount of spherical glass particles was raised higher than $60 \%$-wt, the strength of GIC was found to be weaker. The larger the size of the spherical particle, the smaller the specific surface area was. Therefore, the leaching of $\mathrm{Al}$ or other metal ions from the glass powder was much less, resulting in less crosslinking in cement formation, therefore lowering the mechanical strength of GICs ${ }^{6}$. In addition, the ultimate strength was found to increase to $24 \%$ from $111.93 \pm 10.81$ $\mathrm{MPa}$, observed for the original glass containing $60 \%$-wt spherical powder. These results confirm the improved cement strength by pre-reaction of glass in combination with using the optimized portion of spherical glass particles at $60 \%$-wt.

\section{DISCUSSION}

In this study, the GICs containing pre-reacted glass in the form of spherical powder were prepared. The phase, composition and shape of the glass powder were investigated for their improving effects on the mechanical strength of GICs.

The study by Prosser et $a l .{ }^{9)}$ reported that the presence of crystallites in the glass such as corundum, rutile, aluminum titanate and baddeleyite increased the flexural strength of the cement. The phase-separated glasses were also reported to enhance the compressive strength of the cements compared to single-phase glasses ${ }^{7}$. Thus, the phase-separated opal glass containing crystallites of baddeleyite, $\mathrm{ZrO}_{2}$, developed in this work could tentatively enhance the mechanical strength of the cement.

The hardening reaction of GICs was an acid-base reaction between the carboxyl groups of the polymeric acid and $\mathrm{Al}^{3+}$ and $\mathrm{Ca}^{2+}$ ions released from the glass powder as determined by several techniques such as Raman, FTIR and NMR spectroscopy ${ }^{26-28)}$. In this work, FTIR results showed the increasing of the extent of acid-base reaction and bound water as amount of Fuji II liquid increased. The poorer cement strength was observed upon increasing amount of Fuji II liquid. The weaker strength was a result of the presence of dissoluble ions due to highly moistened glass upon increasing the pre acid-base reaction extent ${ }^{29-32}$. This ion dissoluble effect overcame the strengthening of the cement induced by the pre-reacted glass. As a result, the pre-reacted glass produced from $2 \%$-wt Fuji II liquid, which had the least pre acid-base reaction extent, exhibited the highest strength compared to other pre-reacted glasses and the original glass. This improved mechanical strength of the pre-reacted glass produced from $2 \%$-wt Fuji II liquid was in consistent with the studies by Xu et al. ${ }^{19)}$, Ikemura et $a l .{ }^{20,21)}$ and Robert et al. ${ }^{22)}$

Spherical glass particle produced by flame spraying and inductively coupled radio frequency plasma spraying techniques was suggested to improve mechanical strength of the cement ${ }^{6)}$. However, the more the dense spherical glass particle, the lower the strength values of GICs was obtained. The lack of areas for acid to attack the glass structure in dense particles resulted in a small amount of released ion which acted as cross-linking sites in cement forming. Spray drying technique produced less dense spherical particles by forming loosely agglomerated glass. The obtained spherical particle promoted flow ability during cement mixing, resulting in a decrease in the viscosity of cement which eased cement handling while mixing. The loose agglomeration of glass also benefited the susceptibility of acid to attack on the glass surface, causing extensive cement formation and superior cement hardening.

The influences of particle size and particle shape on cement strength were also investigated in this work by using different mixing ratios between spherical and irregular shaped glass powder. Bimodal particle size distributions likely induced a high mechanical strength because of the high packing density of glass ionomer cements $^{6}$. . The larger glass particles obtain the lower specific surface area which reduced the chance for $\mathrm{Al}$ ions to form cross-linking cement, resulting in weak GICs. Meanwhile, the fine particles have a large specific surface area leading to the accelerating of the setting time and inducing a high degree of cross-linking ${ }^{10}$. In this study, the spherical and irregular shaped glass particles were the representatives of coarse and fine particles, respectively. The optimum cement strength around $140 \mathrm{MPa}$ was obtained from the glass with a mixing ratio of spherical to irregular shaped glass powder at $60: 40$ by weight. Similarly, the work by Okabayashi et $a l .{ }^{10)}$ suggested that the glass should consist of $50-95 \%$ of the coarse particles and $5-50 \%$ of the fine particles by weight.

\section{CONCLUSION}

Spherical-shaped glass powder can be successfully produced through the spray drying method. The pre acid-base reaction as well as the use of mixed particles including spherical and irregular particles is essential in glass powder preparation to enhance the glass ionomer cement strength. In this study, the pre-reacted glass with a mixing ratio of spherical to irregular particles at 60:40 by weight showed promising strength for restorative dental application.

\section{ACKNOWLEDGMENT}

This work was performed with the support of the National Metal and Materials Technology Center, the Ministry of Science and Technology of Thailand, Project 
No. MT-B-49-BMD-07-130-I.

\section{REFERENCES}

1) Wilson $\mathrm{AD}$, Kent BE. Surgical cement. United Kingdom Patent No.1,316,129; 1973.

2) Wilson $\mathrm{AD}$, Kent BE. A new translucent cement for dentistry: the glass ionomer cement. Br Dent J 1972; 132: 133-135.

3) Hübel S, Mejàre I. Conventional versus resin-modified glassionomer cement for glass II restorations in primary molars. A 3-year clinical study. Int J Paediat Dent 2003; 13: 2-8.

4) Smith DC. Development of glass-ionomer cement systems. Biomaterials 1998; 19: 467-478.

5) Cho SY,Cheng AC. A review of glass ionomer restorations in the primary dentition. J Can Dent Assoc 1999; 65: 491-495.

6) Gu YW, Yap AUJ, Cheang P, Kumar R. Spheroidization of glass powders for glass ionomer cements. Biomaterials 2004; 25: 4029-4035.

7) Nicholson JW. Chemistry of glass-ionomer cements: a review. Biomaterials 1998; 19: 485-494.

8) Bertolini MJ, Zaghete MA, Gimenes R, Freitas de Souza R, Vaz LG. Preparation of new glass systems by the polymeric precursor method for dental applications. J Non-Cryst Solids 2004; 344: 170-175.

9) Prosser HJ, Powis DR, Wilson AD. Glass-ionomer cements of improved flexural strength. J Dent Res 1986; 65: 146-148.

10) Okabayashi M, Ohno H, Kusumoto K. Glass-ionomer cement composition, United Kingdom Patent Application No. 2,215,728(A); 1989.

11) Hirasawa M, Kato S, Takuno M. Glass powder for glass ionomer cement. Japan Patent No. 2003-095837; 2003.

12) Griffin SG, Hill RG. Influence of glass composition on the properties of glass polyalkenoate cements. Part I: influence of aluminum to silicon ratio. Biomaterials 1999; 20: 1579-1586.

13) Kent BE, Lewis BG, Wilson A. Glass ionomer cement formulations: I. The preparation of novel fluoroaluminosilicate glasses high in fluorine. J Dent Res 1979; 58: 1607-1619.

14) Akahane S, Hirota K, Tomioka K. Fluoroaluminosilicate glass powder for dental glass ionomer cement. United Kingdom Patent Application No. 2,180,833(A); 1987.

15) Moshaverinia A, Ansari S, Moshaverinia M, Roohpour N, Darr JA, Rehman I. Effects of incorporation of hydroxyapatite and fluoroapatite nanobioceramics into conventional glass ionomer cements (GIC). Acta Biomater 2008; 4: 432-440.

16) Vehring R, Foss WR, Lechuga-Ballesteros D. Particle formation in spray drying. Aerosol Sci 2007; 38: 728-746.

17) Hertzberg RW. Deformation and fracture mechanics of engineering materials. New York: Wiley; 1996.

18) Crisp S, Wilson AD. Reactions in glass ionomer cements: I.
Decomposition of the powder. J Dent Res 1974; 53: 1408-1412.

19) $\mathrm{Xu}$ HHK, Eichmiller FC, Antonucci JM, Schumacher GE, Ives LK. Dental resin composites containing ceramic whiskers and precured glass ionomer particles. Dent Mater 2000; 16: 356-363.

20) Ikemura K, Tay FR, Kouro Y, Endo T, Yoshiyama M, Pashley DH. Optimizing filler content in an adhesive system containing pre-reacted glass-ionomer fillers. Dent Mater 2003; 19: 137-146.

21) Ikemura K, Tay FR, Endo T, Pashley DH. A review of chemical-approach and ultramorphological studies on the development of fluoride-releasing dental adhesive comprising new pre-reacted glass ionomer (PRG) fillers. Dent Mater J 2008; 27: 315-339.

22) Robert TA, Miyai K, Ikemura K, Fuchigami K, Kitamura T. Fluoride ion sustained release preformed glass ionomer filler and dental compositions containing the same. United States Patent No. 5,883,153; 1999.

23) Hurrell-Gillingham K, Reaney IM, Miller CA, Crawford A, Hatton PV. Devitrification of ionomer glass and its effect on the in vitro biocompatibility of glass-ionomer cements. Biomaterials 2003; 24: 3153-3160.

24) International Organization for Standardization (ISO) Technical Committee (2007E). Dentistry-water-based cements-Part 1: Powder/liquid acid-base cements ISO 9917-1. Geneva, Switzerland.

25) Randklev RM. Glass ionomer cement powder. United States Patent No. 4,808,228; 1989.

26) Matsuya S, Maeda T, Ohta M. IR and NMR analyses of hardening and maturation of glass-ionomer cement. J Dent Res 1996; 75: 1920-1927.

27) Young AM. FTIR investigation of polymerisation and polyacid neutralisation kinetics in resin-modified glass-ionomer dental cements. Biomaterials 2003; 23: 3289-3295.

28) Young AM, Rafeeka SA. Howlett JA. FTIR investigation of monomer polymerisation and polyacid neutralisation kinetics and mechanisms in various aesthetic dental restorative materials. Biomaterials 2004; 25: 823-833.

29) Mojon P, Kaltio R, Feduik D, Hawbolt EB, MacEntee MI. Short-term contamination of luting cements by water and saliva. Dent Mater 1996; 12: 83-87.

30) Negm MN, Beech DR, Grant AA. An evaluation of mechanical and adhesive properties of polycarboxylate and glass ionomer cements. J Oral Rehabil 1982; 9: 161-167.

31) Wasson EA, Nicholson JW. New aspect of the setting of glassionomer cements. J Dent Res 1993; 72: 481-483.

32) Mesu FP. Degradation of luting cements measured in vitro. J Dent Res 1982; 61: 665-672. 